\title{
An observational retrospective analysis of the main metastatic site and corresponding locoregional treatment as a prognostic factor in metastatic gastric cancer
}

\author{
RICCARDO GIAMPIERI $^{1,2}$, LUCA CANTINI ${ }^{1}$, MICHELA DEL PRETE ${ }^{3}$, \\ ALESSANDRO BITTONI $^{2}$, ENRICA GIGLIO ${ }^{1}$, ALESSANDRA MANDOLESI ${ }^{4}$, \\ ELENA MACCARONI $^{2}$, ANDREA LANESE ${ }^{2}$, TANIA MELETANI ${ }^{1}$, MARIA GIUDITTA BALEANI $^{1}$, \\ RENATO BISONNI $^{3}$, MARINA SCARPELLI ${ }^{4}$ and ROSSANA BERARDI ${ }^{1,2}$ \\ ${ }^{1}$ Clinical Oncology, Department of Clinical and Molecular Sciences, Marche Polytechnic University; \\ ${ }^{2}$ Clinical Oncology, Ospedali Riuniti Ancona, I-60126 Ancona; ${ }^{3}$ Medical Oncology, Azienda \\ Ospedaliera di Fermo, I-63900 Fermo; ${ }^{4}$ Pathology, Department of Biomedical Sciences \\ and Public Health, Marche Polytechnic University, I-60126 Ancona, Italy
}

Received March 17, 2020; Accepted November 18, 2020

DOI: $10.3892 / 01.2021 .12528$

\begin{abstract}
Despite novel drugs, the prognosis for patients with metastatic gastric cancer remains poor. In rare instances, locoregional therapies are used in addition to standard chemotherapy in patients with oligometastatic involvement. This type of approach has not been supported by solid published evidence. The aim of the present retrospective study was to assess the prognostic impact of factors such as metastatic site, tumour histology and locoregional treatment in patients with metastatic gastric cancer. A total of 184 patients with metastatic gastric or gastroesophageal junction adenocarcinoma who received at least one line of palliative therapy with doublet or triplet chemotherapy were enrolled in the current analysis. Median overall survival (OS) was 8.32 months (95\% CI, 7.02-9.41) and median progression-free survival (PFS) was 4.16 months (95\% CI, 3.24-5.08). Lung metastases vs. other sites of metastatic involvement [hazard ratio (HR), 0.27; $\mathrm{P}=0.0133$ ] and intestinal histology $(\mathrm{HR}, 0.48 ; \mathrm{P}=0.08)$ were significantly associated with an improved OS. Improved PFS was also observed (HR, $0.49 ; \mathrm{P}=0.10$ and $\mathrm{HR}, 0.72 ; \mathrm{P}=0.08$ for lung metastases and intestinal histology, respectively). Second line chemotherapy and locoregional treatment of metastases
\end{abstract}

Correspondence to: Professor Riccardo Giampieri, Clinical Oncology, Department of Clinical and Molecular Sciences, Marche Polytechnic University, Via Conca 71, I-60126 Ancona, Italy E-mail: r.giampieri@staff.univpm.it

Abbreviations: OS, overall survival; PFS, progression-free survival; DCR, disease control rate; SD, stable disease; PR, partial response; $\mathrm{CR}$, complete response

Key words: gastric cancer, locoregional, metastasis, lung, chemotherapy, surgery, radiotherapy (surgery or radiotherapy) were associated with improved OS (HR, 0.52; $\mathrm{P}<0.0001$ and $\mathrm{HR}, 0.35 ; \mathrm{P}<0.0001$, respectively). Multivariate analysis confirmed an independent prognostic role for OS only for locoregional treatment, second line treatment and intestinal histology. The present results suggested that the presence of lung metastases alone was not a relevant prognostic factor and was influenced by the availability of further lines of treatment or by locoregional treatments. Locoregional treatments in patients with oligometastatic disease should be offered as they allow prolonged survival in patients with otherwise relatively short life expectancy.

\section{Introduction}

Gastric cancer remains a major health problem worldwide; the most recent worldwide epidemiological data suggest that its incidence and mortality among the most common types of solid tumour are at fifth and third place, respectively (1). Altogether, 783,000 patients died due to gastric cancer in 2018 (1). Even though this type of cancer has been subject to a lot of research, in the last decades its prognosis has not improved significantly. The estimated median overall survival (OS) is usually 10-12 months in metastatic patients with HER-2 negative disease (2). In this group of patients, palliative chemotherapy represents the mainstay of treatment (3).

New targeted therapies have recently demonstrated improvements in terms of patient outcomes. Trastuzumab (4) and Ramucirumab $(5,6)$ have shown significant benefits to survival and thus are widely used in Western countries. Apatinib, which is currently under development, is exhibiting signs of being a promising agent (7), whereas other drugs such as Regorafenib are still under investigation or have yielded disappointing results (such as in the case of Pertuzumab) $(8,9)$.

Another therapeutic approach that should be considered is surgery. The real benefit of surgical resection of the primary tumour in patients with metastatic disease remains unclear. The only available randomized trial (REGATTA) has not revealed 
any survival benefit of gastrectomy followed by chemotherapy compared with chemotherapy alone in 175 patients with advanced gastric cancer with a single non-curable site of disease confined to either the liver, peritoneum or para-aortic lymph nodes (10). Conversely, previous retrospective data (11-14) and meta-analyses (15) have suggested that surgery of the primary tumour significantly prolongs survival, increasing the median OS time to 14.9 months in patients with palliative gastrectomy, regardless of metastatic site. An even greater survival advantage has been observed in highly selected patients with synchronous distant metastases who had undergone both gastrectomy and metastasectomy, with a median OS time of 21.9 months (16). The resection of sites of metastatic involvement with a different likelihood of obtaining radical surgery (hepatic, peritoneal or distant lymph nodes) has been associated with an increase in survival from 1.3 to 5 years (17). Prolonged survival has been also achieved in patients who have been randomized to maximal cytoreductive surgery combined with regional heated intraperitoneal and systemic chemotherapy, as shown by the GYMMSA trial (18).

However, the aforementioned retrospective data cannot be considered conclusive due to selection bias. In fact, only patients with good performance status, a more limited disease and a tumour biology favouring slow tumour growth and selective metastatic spread $(19,20)$ could have been scheduled for surgery. Furthermore, therapies administered before and after surgery may represent a confounding factor. Results have shown that palliative chemotherapy combined with gastrectomy may determine a survival benefit compared with palliative gastrectomy alone (15).

Notably, albeit scarce data concerning locoregional treatment of patients with gastric cancer have been published, only a few retrospectively collected case series for patients with isolated lung metastases due to gastric cancer can be found in published literature $(21,22)$. About $15 \%$ of patients with advanced disease present metastases to lungs, and regardless of the treatment received (either chemotherapy or best supportive care), they seem to have an improved OS compared with other types of metastatic spread (23).

In other gastrointestinal malignancies, such as in colorectal cancer, there is published evidence that supports locoregional management of lung metastases (24). However, there are no published prospective randomized studies on this matter (the PULMICC trial is now ongoing) (25). Nonetheless, despite a lack of proper clinical trials, surgical resection of lung metastases is performed currently and safely in everyday clinical practice. The 5-year survival rates of patients undergoing this procedure are 30-50\%, which are comparable to those observed in patients who undergo liver resection (26). A few prognostic indicators that may affect the outcome have been identified, including number of metastases, disease-free interval between the primary tumour and the lung recurrence, and hilar/mediastinal lymph node involvement (24-26).

There is a lack of similar evidence in metastatic gastric cancer, mostly due to the relatively small amount of available data and the worse prognosis of this disease compared with colorectal cancer. In addition, lung metastases are frequently removed at the same time as liver metastases, according to a population-based review (23), thus decreasing the number of patients that would ultimately be candidates to receive radical surgery.

The present study aimed to determine the role of several prognostic factors, highlighting the differences among patients according to metastatic site, tumour histology and treatment received (either systemic or local). Wider knowledge of mechanisms involved in metastatic gastric cancer development and metastatic spread, in light of the new molecular classification (27), may strengthen the rationale behind lung metastasectomy and ensure a more tailored and evidence-based approach for these patients.

\section{Materials and methods}

Patient selection and main stratification factors. A total of 184 patients with histologically confirmed metastatic gastric or gastroesophageal junction adenocarcinoma were considered eligible for analysis. Patients should have received $\geq 1$ line of chemotherapy for metastatic disease with doublet or triplet chemotherapy (either combinations with cisplatin or oxaliplatin, or combinations with 5-fluorouracil or capecitabine were admitted). Patients who received prior surgery for locally advanced disease and who received adjuvant chemotherapy were admitted into the analysis if $>6$ months had elapsed between the end of adjuvant chemotherapy and the first radiological sign of disease relapse.

In addition to sex, age at diagnosis, performance status at the start of palliative chemotherapy and previous adjuvant chemotherapy, the main stratification factors were disease histotype by Lauren classification $(28,29)$ (intestinal, diffuse, signet ring cell or not otherwise specified), primary sites of metastatic involvement (lung only, liver only, other sites with the addition of peritoneal involvement or other sites of metastatic spread without peritoneal involvement), palliative surgery for the primary tumour (yes or no) and timing of metastatic involvement (synchronous or metachronous). The impact of age at the start of first-line treatment on survival outcomes was assessed by using two different clinically chosen cut-off values, $<75$ or $\geq 75$ years, and $\leq 40$ or $>40$ years. Whether patients had received second-line treatment or not was used as a stratification factor only for OS. Consecutive patients treated at Azienda Ospedaliera Universitaria Ospedali Riuniti Ancona (Ancona, Italy) between January 1999 and June 2017 were included in the present study.

Statistical analysis. The aim of the analysis was to assess whether one or more of the aforementioned stratification factors may have an impact on patient prognosis. Survival outcomes and response to first-line treatment were retrospectively collected for all patients included in the analysis. $\mathrm{P}<0.05$ was considered to indicate a statistically significant difference.

OS time was calculated as the time interval between the start of first-line chemotherapy and the time of death due to any cause or last follow-up visit. Progression-free survival (PFS) time was calculated as the time interval between the start of first-line chemotherapy and the time of death or of the first radiological or clinically meaningful sign of disease progression (whichever came first). Survival analysis was 
Table I. Baseline tumour and patient characteristics $(n=184)$.

Characteristic Value Percentage, $\%$

Median age (range), years

$63(25-83)$

Sex, n

Male

Female

ECOG PS, n

0

1

Resection of primary tumour, $n$

Yes

No

Adjuvant chemotherapy, $\mathrm{n}$

Yes

No

119

65

110

74

95

89

41

143

Neoadjuvant chemotherapy, $\mathrm{n}$

Yes

No

Histological subtype, $\mathrm{n}$

Intestinal

Diffuse

Signet ring cells, $\mathrm{n}$

Other

Unknown

HER-2 status, $\mathrm{n}$

Positive

Negative

Not assessed

Second-line chemotherapy, $\mathrm{n}$

Yes

No

Timing of metastases

presentation, $\mathrm{n}$

Synchronous

Metachronous

Site of metastatic

involvement, $\mathrm{n}$

Lung only

Liver only

Lymph nodes only

Peritoneal only

Bone only

Other sites with peritoneal

involvement

Other sites without peritoneal

involvement

Locoregional treatment, $\mathrm{n}$

Yes

No

Ethnicity, n

White Caucasian

Hispanic
Table I. Continued.

\begin{tabular}{lcc}
\hline Characteristic & Value & Percentage, $\%$ \\
\hline Age $\geq 75$ years old, $\mathrm{n}$ & & \\
Yes & 29 & 16 \\
No & 155 & 84 \\
Age $\leq 40$ years old, $\mathrm{n}$ & & \\
Yes & 14 & 8 \\
No & 170 & 92 \\
Response to first line & & \\
chemotherapy & & \\
Complete response & 10 & 6 \\
Partial response & 42 & 24 \\
Stable disease & 37 & 21 \\
Progressive disease & 86 & 49 \\
Not evaluable & 9 & 5 \\
\end{tabular}

ECOG PS, Eastern Cooperative Oncology Group performance status.

calculated using the Kaplan-Meier method and differences among stratifying factors were assessed by log-rank test. Multivariate analysis was conducted by Cox-proportional hazards regression.

Response rates were defined according to the Response Evaluation Criteria In Solid Tumors (RECIST) 1.1 (30). Patients, as per standard clinical practice, had received chest-abdomen CT scans once every 3 months in order to evaluate response to treatment. Disease control rate (DCR) was defined as the sum of patients who had stable disease (SD), partial response (PR) or complete response (CR). For patients with non-measurable disease according to RECIST, only survival outcomes were assessed. The association among categorical variables was assessed by Fisher's exact test for binomial categorical variables and by $\chi^{2}$ test in all other instances. The present study was reviewed by a biomedical statistician. All statistical analyses were performed using MedCalc Statistical Software version 19.2.1 (MedCalc Software bvba) and R software (version 3.6.2; https://www.r-project.org).

\section{Results}

Treatment outcomes in the whole patient population. A total of 184 patients were eligible for analysis. The main stratifying factors of the whole population are shown in Table I. In the whole cohort of patients, median OS time was 8.32 months $(95 \% \mathrm{CI}$, 7.016-9.410), while mean OS time was 19.33 months (95\% CI, 13.39-25.26). Similarly, median PFS time was 4.16 months (95\% CI, 3.24-5.08), while mean PFS time was 7.35 months (95\% CI, 5.22-9.49) (data not shown).

A total of $10(6 \%)$ patients achieved CR, 42 (24\%) achieved PR, 37 (21\%) achieved SD and 86 (49\%) progressed during first-line chemotherapy. A total of $9(5 \%)$ patients were not assessed for response by RECIST due to a lack of target lesions (as they were affected by bone or peritoneal metastases) (Table I). A total of 7/184 (4\%) patients had only lung metastases, 41/184 (22\%) had only liver metastases, 12/184 

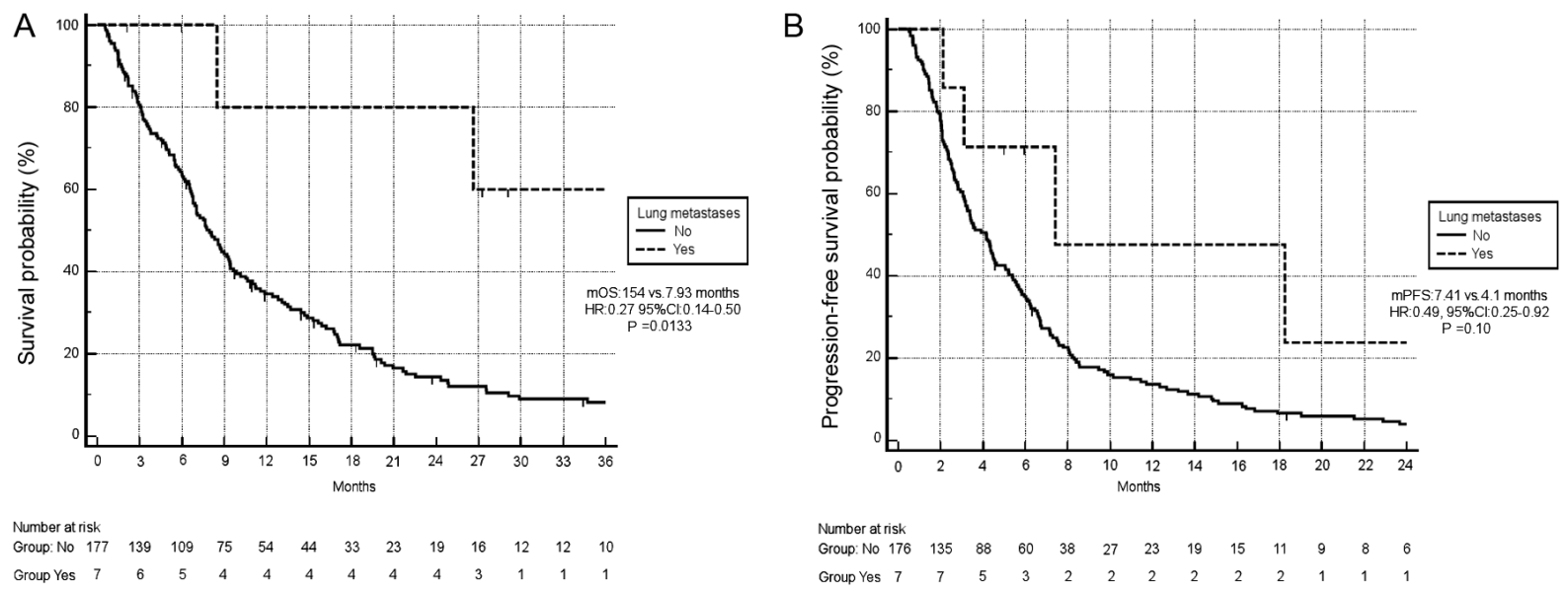

Figure 1. Survival outcomes in patients stratified by lung metastases. (A) OS curves and (B) first-line PFS curves for lung metastases vs. no lung metastases. HR, hazard ratio; m, median.

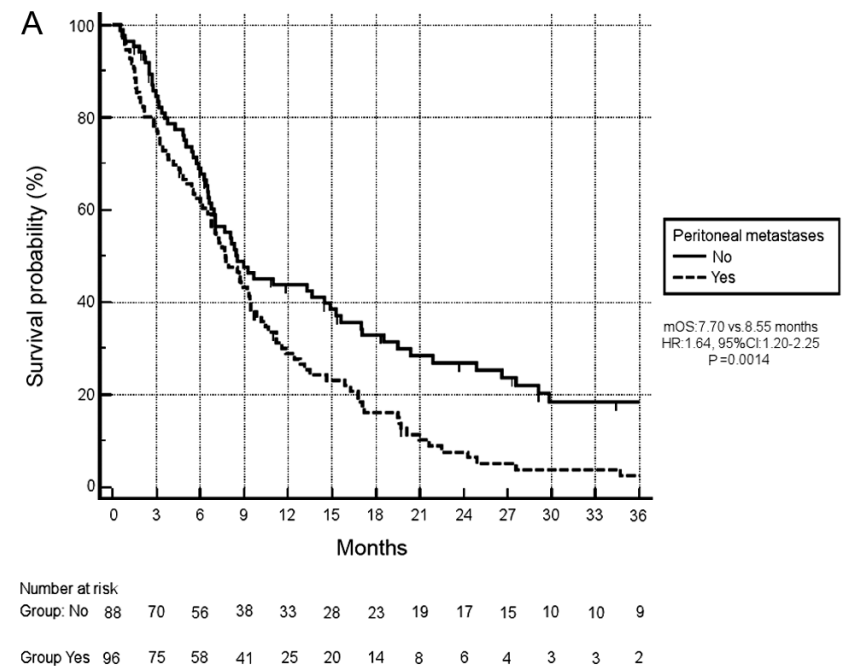

Figure 2. Survival outcomes in patients stratified by peritoneal metastases. peritoneal metastases. HR, hazard ratio; m, median.

(6\%) patients had only metastases in distant lymph nodes (either abdominal or thorax lymph nodes), 68/184 (37\%) had only peritoneal involvement and 52/184 (28\%) patients had metastases in multiple organs. The remaining $4 / 184(2 \%)$ had only bone metastases (Table I).

Treatment outcomes stratified by metastatic site. A statistically significant association between different sites of metastatic involvement and both OS ( $\mathrm{P}=0.003)$ and $\mathrm{PFS}(\mathrm{P}=0.0018)$ was observed. When comparing lung metastases only vs. other sites of metastatic involvement, significantly improved OS was observed in the former group [median OS, 154 months vs. 7.93 months, respectively; hazard ratio (HR), 0.27; $95 \%$ CI, 0.14-0.50; P=0.0133; Fig. 1A]. Similarly, there was a trend towards improved PFS in patients with lung metastases compared with other sites of metastatic involvement (median PFS, 7.4 months vs. 4.1 months, respectively; HR, 0.49; 95\% CI, 0.25-0.92; $\mathrm{P}=0.10$; Fig. 1B). Similarly, response rates in patients with only lung metastases (7/184) were significantly improved compared with those in patients with other sites of

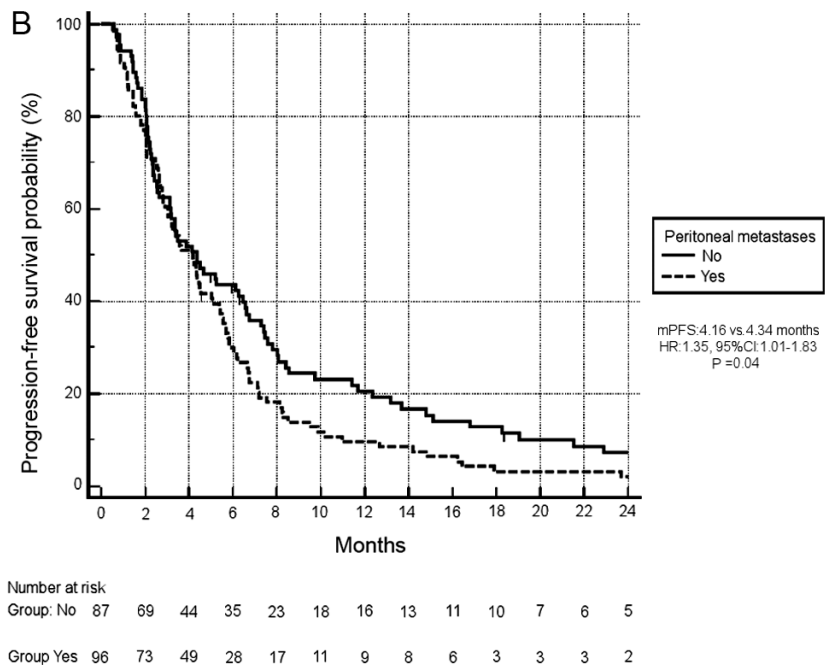

metastatic involvement $(6 / 7,85 \%$ vs. $46 / 177,26 \%$; $\mathrm{P}=0.0022$; data not shown). Conversely, peritoneal metastases had an unfavourable impact on survival: Median OS time for patients with peritoneal metastases $(96 / 184,52 \%)$ was 7.70 months vs. 8.55 months, respectively (HR, 1.64; 95\% CI, 1.20-2.25; $\mathrm{P}=0.0014 ;$ Fig. 2A). In addition, patients with peritoneal metastases had a significantly worse median PFS time (median PFS, 4.16 months vs. 4.34 months, respectively; HR, 1.35; 95\% CI, 1.01-1.83; P=0.04; Fig. 2B).

When comparing the OS of patients stratified by single-organ involvement, lung metastases were associated with significantly improved prognosis (HR, 0.39 and $95 \%$ CI, 0.20-0.77 for lung vs. liver; HR, 0.33 and $95 \%$ CI, 0.14-0.75 for lung vs. lymph nodes; HR, 0.23 and $95 \%$ CI, $0.11-0.44$ for lung vs. peritoneum; and HR, 0.22 and $95 \% \mathrm{CI}, 0.12-0.45$ for lung vs. multiple sites). On the other hand, liver metastases were not associated with differences in survival compared with lymph node metastases (HR, 0.84 and 95\% CI, 0.45-1.57), while survival was significantly improved compared with either peritoneal metastases (HR, 0.58 and 95\% CI, 

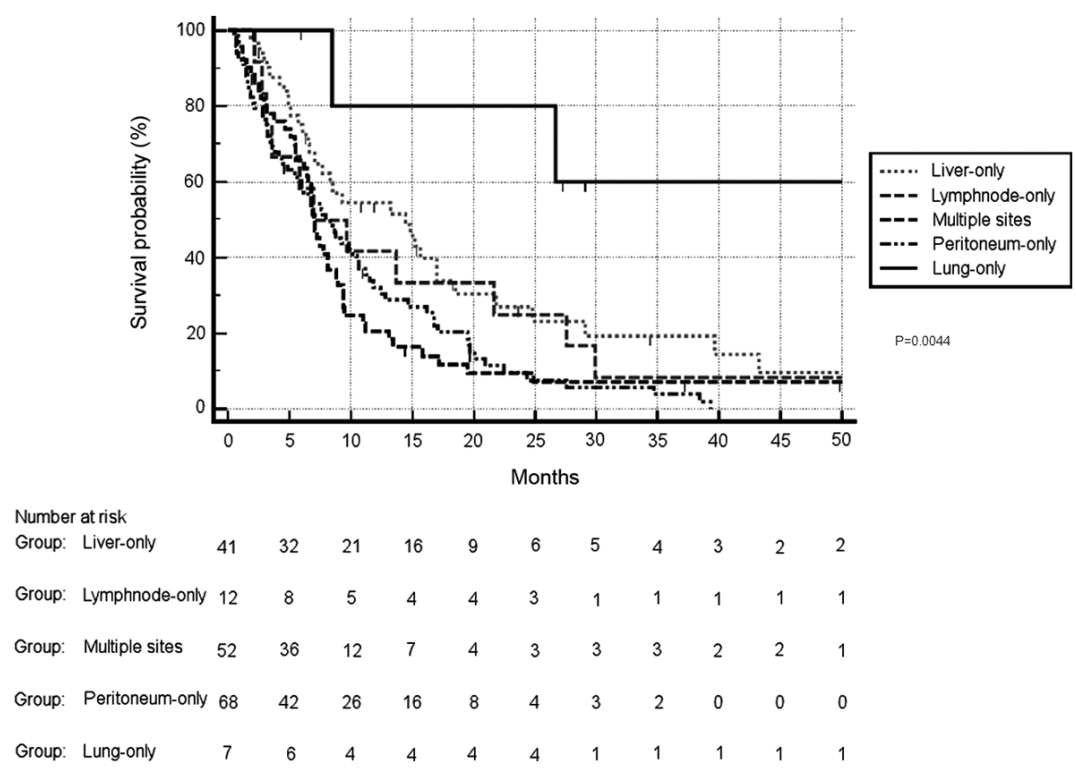

Figure 3. Overall survival curves according to site of metastatic involvement.

A

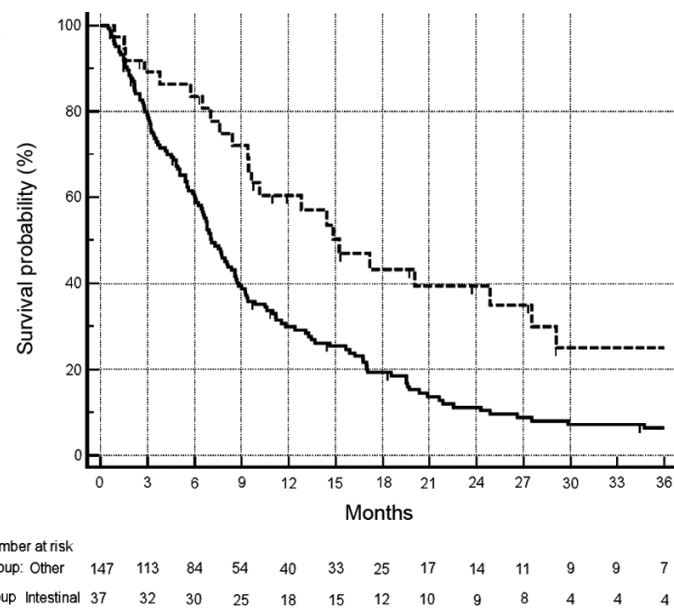

B

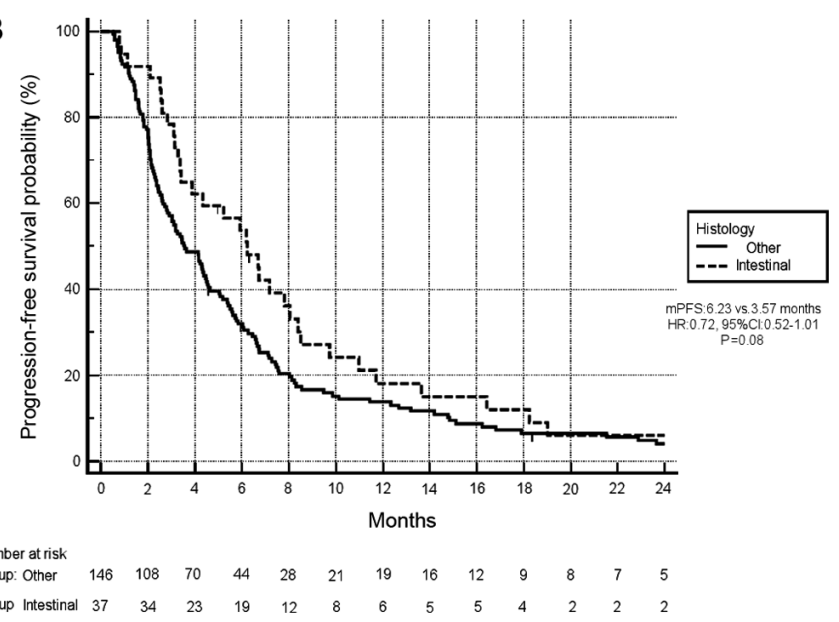

Figure 4. Survival outcomes in patients stratified by histology. (A) OS curves and (B) first-line PFS curves for intestinal histology vs. other histologies. HR, hazard ratio; m, median.

$0.39-0.88$ ) or multiple metastases (HR, 0.56 and $95 \% \mathrm{CI}$, 0.36-0.87). Lymph node metastases were not associated with differences in OS compared with peritoneal metastases (HR, 0.66 and $95 \% \mathrm{CI}, 0.38-1.28)$ or multiple sites of metastatic involvement (HR, 0.66 and 95\% CI, 0.35-1.26). Finally, peritoneal metastases were not associated with differences in OS compared with multiple sites of metastatic involvement (HR, 0.95 and 95\% CI, 0.62-1.46) (Fig. 3). Overall, different sites of metastatic involvement were associated with significantly different survival outcomes $(\mathrm{P}=0.0044)$.

Treatment outcomes stratified by other factors, including histology, other additional treatments, second-line chemotherapy and age. Survival analysis exhibited improved OS in patients with the intestinal subtype compared with other histological subtypes. In particular, the median OS time of patients with the intestinal subtype compared with other histotypes was 15.24 months vs 7.08 months, respectively (HR,
0.48; 95\% CI, 0.34-0.68; P=0.0006; Fig. 4A). Additionally, there was a trend towards improved PFS in patients with the intestinal subtype compared with other subtypes (median PFS, 6.23 months vs. 3.57 months, respectively; HR, 0.72; 95\% CI, $0.52-1.01$; $\mathrm{P}=0.08$; Fig. 4B). In terms of response rates, 19/37 (51\%) patients achieved PR or CR in the intestinal subtype group vs. 23/146 (16\%) in the remaining group, and this difference was statistically significant $(\mathrm{P}=0.000017)$ (data not shown).

A total of 20/184 (11\%) patients received locoregional treatment in addition to chemotherapy: 5 patients received liver metastasectomy, 1 patient received stereotactic radiosurgery for an isolated brain metastasis and underwent surgery for a single skin metastasis, 2 patients underwent peritonectomy and hyperthermic intraperitoneal chemotherapy, 2 patients underwent bilateral palliative oophorectomy for ovarian metastases, 1 patient underwent abdominal lymphnodal dissection for para-aortic lymphnodal involvement, 2 patients underwent lung 

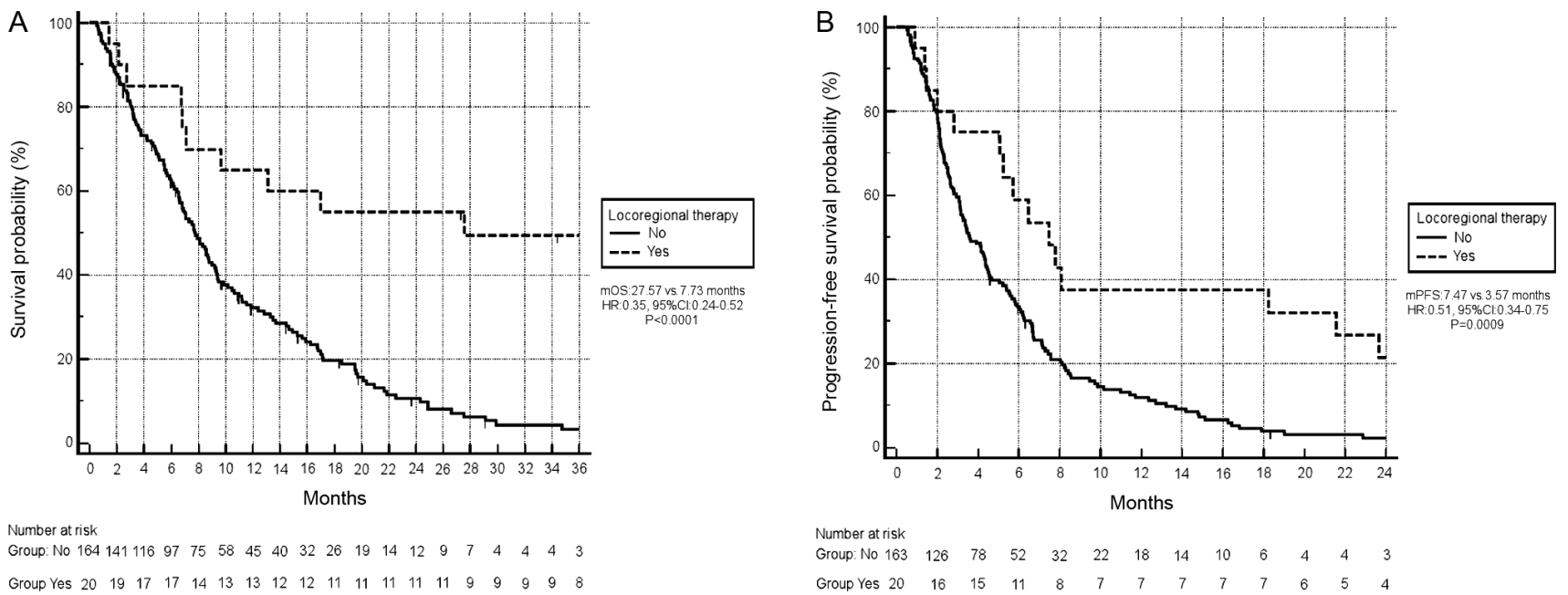

Figure 5. Survival outcomes in patients stratified by having received locoregional therapy. (A) OS curves and (B) first-line PFS curves for locoregional treatment vs. no locoregional therapy. HR, hazard ratio; m, median.

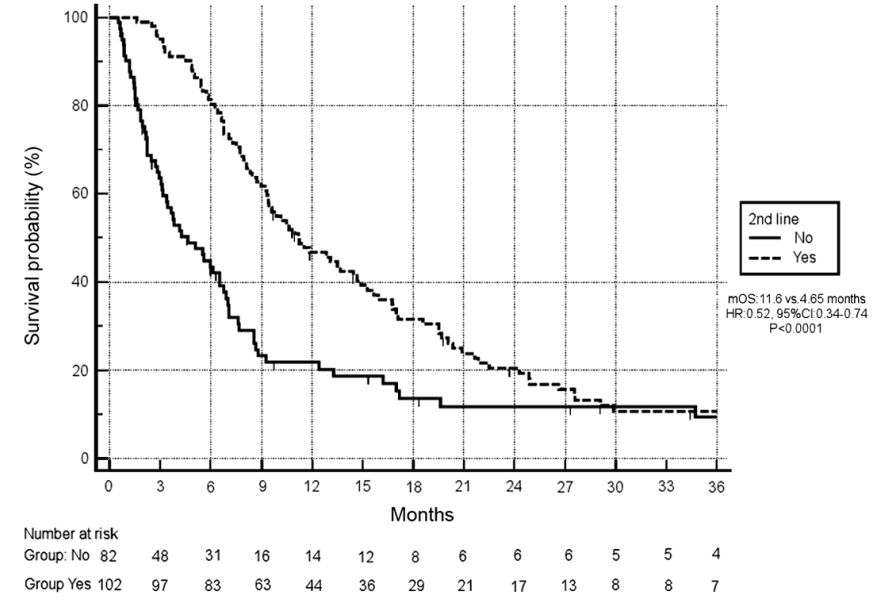

Figure 6. OS curves in patients stratified by having received second-line treatment. HR, hazard ratio; m, median.

resection (and one of them was subsequently submitted to liver surgery for an isolated liver metastasis), 2 patients received stereotactic radiosurgery for lung metastases, 1 patient received radiotherapy in mediastinal lymph nodes and the remaining 4 patients all received radiotherapy for bone metastases.

Survival analysis revealed that locoregional treatment of metastatic sites (either by surgery or radiotherapy) was significantly associated with an improved OS (mOS 27.57 months vs. 7.73 months, respectively; HR, 0.35 ; 95\% CI, 0.24-0.52; $\mathrm{P}<0.0001 ;$ Fig. $5 \mathrm{~A}$; OS curve in patients who also received locoregional treatment of metastatic sites vs. only chemotherapy). Locoregional treatment of metastatic sites was also associated with significantly longer PFS (median PFS 7.47 months vs. 3.57 months, respectively; HR, 0.51; 95\% CI, 0.34-0.75; P=0.0009; Fig. 5B; PFS curve in patients who also received locoregional treatment of metastatic sites vs. only chemotherapy).

A total of $29 / 184(16 \%)$ patients were $\geq 75$ years old. OS was not significantly different compared with younger patients (median OS 7.08 months vs. 8.32 months, respectively;
HR, 0.98; 95\% CI, 0.62-1.53; P=0.93). First-line PFS was also not significantly different (median PFS 4.36 months vs. 4.16 months, respectively; HR, 0.89; 95\% CI, 0.59-1.35; $\mathrm{P}=0.61$ ). A total of $14 / 184$ (8\%) patients were $\leq 40$ years old. OS was not significantly different compared with older patients (median OS 11.83 months vs. 8.13 months, respectively; HR, 0.78; 95\% CI, 0.46-1.34; P=0.38). First-line PFS was also not significantly different (median PFS 2.82 months vs. 4.29 months, respectively; HR, 0.76; 95\% CI, 0.44-1.29; $\mathrm{P}=0.31$ ) (data not shown).

Finally, 82/184 (45\%) patients received second-line therapy after first-line disease progression. A significantly improved OS was observed in this group of patients compared with those without second-line treatment (11.6 months vs. 4.65 months, respectively; HR, 0.52; 95\% CI, 0.37-0.74; $\mathrm{P}<0.0001$; Fig. 6).

Results of multivariate analysis. Results of the multivariate analysis are shown in Table II. The only factors that remained significantly associated with differences in OS were locoregional treatment (HR, 0.35; 95\% CI, 0.19-0.64; $\mathrm{P}=0.007$ ), second-line treatment (HR, 0.50 ; 95\% CI, 0.36-0.69; $\mathrm{P}<0.0001)$ and intestinal histology $(\mathrm{HR}, 0.58 ; 95 \% \mathrm{CI}$, $0.37-0.90 ; \mathrm{P}=0.0152$ ). The only factor that was significantly associated with differences in PFS was locoregional treatment of metastases (HR, 0.45; 95\% CI, 0.27-0.72; $\mathrm{P}=0.0034$ ).

\section{Discussion}

The present analysis was focused on assessing the role of a series of prognostic factors in patients with metastatic gastric cancer. The analysed population was comprised almost entirely of white-Caucasian patients: Since ethnicity is an important factor in gastric cancer, this should be considered when assessing the current results. Tumour histology has been previously described as a strong prognostic factor. A previous study of 248 patients with metastatic gastric cancer stratified by histology demonstrated that patients with the intestinal subtype of gastric cancer had improved PFS and OS compared 
Table II. Summary of results of multivariate analysis.

\begin{tabular}{|c|c|c|c|c|c|c|}
\hline \multirow[b]{2}{*}{ Stratifying factor } & \multicolumn{3}{|c|}{ Progression-free survival } & \multicolumn{3}{|c|}{ Overall survival } \\
\hline & HR & $95 \% \mathrm{CI}$ & P-value & HR & $95 \% \mathrm{CI}$ & P-value \\
\hline Intestinal histology (yes vs. no) & 0.81 & $0.55-1.19$ & 0.277 & 0.58 & $0.37-0.90$ & 0.015 \\
\hline Lung metastases (yes vs. no) & 0.63 & $0.25-1.59$ & 0.328 & 0.49 & $0.15-1.60$ & 0.241 \\
\hline Peritoneal metastases (yes vs. no) & 1.21 & $0.88-1.64$ & 0.210 & 1.35 & $0.97-1.89$ & 0.073 \\
\hline Second-line treatment (yes vs. no) & I & 1 & I & 0.50 & $0.36-0.69$ & $<0.001$ \\
\hline Locoregional treatment (yes vs. no) & 0.45 & $0.27-0.77$ & 0.003 & 0.35 & $0.19-0.64$ & $<0.001$ \\
\hline
\end{tabular}

HR, hazard ratio

with patients with the diffuse subtype (31). Additionally, a recent meta-analysis including 61,468 patients confirmed that the diffuse type of gastric cancer is a poor prognostic factor regardless of the treatment received (32).

Another factor that was associated with improved OS in the present study was whether patients had received second-line treatment. In a recently published paper (33), stratification by several clinical factors, such as performance status, lactate dehydrogenase levels, neutrophil/lymphocytes ratio and first-line PFS time, was able to identify subsets of patients with second-line OS estimates $>7$ months compared with patients with poor prognostic features.

The present survival analysis suggested that different sites of metastatic involvement may be associated with differences in OS: Lung metastases seemed to have the best survival outcomes, while peritoneal metastases were associated with the worst survival outcomes. However, this was disproved by the multivariate analysis; when the site of metastatic involvement was assessed together with tumour histology, second-line treatment and locoregional therapies, it lost its independent prognostic impact. To the best of our knowledge, this is the first time that this has been proven in patients with gastric cancer.

Regarding other gastrointestinal malignancies, there are several studies that have focused on the improved survival of patients affected by lung metastases, stratified by surgical resection. For example, Brandi et al (34) analysed a retrospective series of 151 patients with metastatic colorectal cancer who received liver or lung metastases resection. A total of 20/151 (13\%) patients had lung involvement only, but no statistically significant differences in survival outcomes were observed in patients with lung metastases compared with liver metastases; the only factors that influenced survival in the multivariate analysis were adjuvant chemotherapy and disease-free interval (34). Gonzalez et al (26) reported survival outcomes of patients who underwent lung metastasectomy for metachronous lung metastases in patients with colorectal cancer previously treated with surgery for liver metastases. Their results revealed a median disease-free survival time after pulmonary metastasectomy of 13 months and that patients who benefited the most from surgery were those with single lung metastases, compared with those having $>1$ site of lung involvement (26).

Regarding gastric cancer, the published data are even scarcer. Iijima et al (21) have analysed the role of lung metastasectomy in patients who have lung metastases as the only site of involvement for gastric cancer. In the 10 patients who were eligible for the analysis, the 3-year OS rate was $30 \%$ (21). In another study by Yoshida et al (22), 10 patients with metastatic gastric cancer were submitted to surgery for solitary lung metastases, with relatively favourable results (75\% 4-year survival rate), as in the present analysis. In a review by Aurello et al (35), 10 published papers including a total of 44 patients with metastatic gastric cancer were described. The median disease-free interval of the resected patients was 35 months and among them, 38 patients had single lung metastases, whereas 6 patients presented with $>1$ lesion (28). Median OS time after resection was 45 months, with a median disease-free survival time of 9 months (35).

Finally, in a large registry based observational retrospective analysis by Shiono et al (36), comprising 3,831 patients who underwent surgical resection for lung metastases arising from different types of cancer, 51 patients had primary gastric cancer.In these patients, survival outcomes were less favourable than in the present analysis, with a 5-year OS rate after resection of $28 \%$, a median survival time of 29 months and median time to recurrence after lung resection of 6 months (36). Even if these results seem to be less optimistic than the present data, it should be considered that these survival rates are better than what is currently achieved just through the use of standard palliative chemotherapy (usually with a median OS time of 12-16 months $(2,37)$. In addition, in the population of patients with disease-free intervals $>12$ months, a statistically significant impact on improved OS was observed, with the 5-year OS rate rising up to $31 \%$ (36).

In the present study, different types of lung metastatic involvement were analysed. In particular, the shortest survival times were observed for patients with diffuse lung involvement, a rarely described phenomenon called Bard's syndrome (caused by diffuse lymphangitic involvement of the whole lung with severe respiratory impairment and imminent risk of death) (38). Similarly to the aforementioned studies, improved survival was observed for patients submitted to surgery with the presence of solitary lung metastases, with sufficiently long ( $>12$ months) relapse-free survival times following the resection of the primary tumour. Additionally, the present study observed a particularly favourable prognosis in one patient who, although not submitted to surgery, received high dose stereotactic radiotherapy of a solitary lesion. 
Locoregional treatments in patients with metastatic gastric cancer, although not recommended for the majority of patients, should be at least offered in selected cases with favourable prognostic features, such as a single site of metastatic involvement, positive probability of achieving a R0 resection and sufficiently prolonged observation time following surgery for the primary tumour (17). A recently published meta-analysis by Gadde et al (17), focusing solely on patients with metastatic gastric cancer amenable to surgery regardless of their site of involvement, revealed significant survival advantages in patients who received surgery, and this improvement in survival was significantly higher when looking at the 1-year time point (with a decreased impact over the course of the following years of observation).

In conclusion, the present data contributes to the body of evidence on patients with oligometastatic gastric cancer, suggesting that, in a few selected cases (those with a relatively favourable first-line PFS) locoregional treatment of isolated metastatic sites should be offered, as it allows for increased survival that would otherwise not be achieved with any other type of medical treatment.

\section{Acknowledgements}

The authors would like to thank Dr Andrew Davies Burd for English revision of this manuscript.

\section{Funding}

The present study was funded by the Università Politecnica delle Marche (Ancona, Italy).

\section{Availability of data and materials}

The datasets used and/or analysed during the current study are available from the corresponding author on reasonable request.

\section{Authors' contributions}

RG, LC, MDP, EG and RBe conducted the present study. EG, LC, MDP, RBi, TM, MGB and AM collected the clinical data. RG, AL and EM performed the statistical analyses. RG, TM, MGB, AM, EG, LC, AB, EM, RBe, MS and RBi participated in the study design and concept. All authors oversaw the drafting of the manuscript, and read and approved the final manuscript.

\section{Ethics approval and consent to participate}

The present study was approved by the Regional Ethical Committee of Marche, Azienda Ospedaliero-Universitaria Ospedali Riuniti of Ancona (Ancona, Italy). All patients provided written informed consent to the research and to all the diagnostic-therapeutic procedures.

\section{Patient consent for publication}

Not applicable.

\section{Competing interests}

The authors declare that they have no competing interests.

\section{References}

1. Bray F, Ferlay J, Soerjomataran I, Siegel RL, Torre LA and Jemal A: Global cancer statistics 2018: GLOBOCAN estimates of incidence and mortality worldwide for 36 cancers in 185 countries. CA Cancer J Clin 68: 394-424, 2018.

2. Cunningham D, Starling N, Rao S, Iveson T, Nicolson M, Coxon F, Middleton G, Daniel F, Oates J and Norman AR: Capecitabine and oxaliplatin for advanced esophagogastric cancer. N Engl J Med 358: 36-46, 2008.

3. Wagner AD, Grothe W, Haerting J, Kleber G, Grothey A and Fleig WE: Chemotherapy in advanced gastric cancer: A systematic review and meta-analysis based on aggregate data. J Clin Oncol 24: 2903-2909, 2006.

4. Bang YJ, Van Cutsem E, Feyereislova A, Chung HC, Shen L, Sawaki A, Lordick F, Ohtsu A, Omuro Y, Satoh T, et al: Trastuzumab in combination with chemotherapy versus chemotherapy alone for treatment of HER2-positive advanced gastric or gastro-oesophageal junction cancer (ToGA): A phase 3, open-label, randomised controlled trial. Lancet 376: 687-697, 2010.

5. Fuchs CS, Tomasek J, Yong CJ, Dumitru F, Passalacqua R, Goswami C, Safran H, Dos Santos LV, Aprile G, Ferry DR, et al: REGARD Trial Investigators. Ramucirumab monotherapy for previously treated advanced gastric or gastro-oesophageal junction adenocarcinoma (REGARD): An international, randomised, multicentre, placebo-controlled, phase 3 trial. Lancet 383: 31-39, 2014.

6. Wilke H, Muro K, Van Cutsem E, Oh SC, Bodoky G, Shimada Y, Hironaka S, Sugimoto N, Lipatov O, Kim TY, et al: Ramucirumab plus paclitaxel versus placebo plus paclitaxel in patients with previously treated advanced gastric or gastro-oesophageal junction adenocarcinoma (RAINBOW): A double-blind, randomised phase 3 trial. Lancet Oncol 15: 1224-1235, 2014.

7. Li J, Qin S, Xu J, Xiong J, Wu C, Bai Y, Liu W, Tong J, Liu Y, $\mathrm{Xu}$ R, et al: Randomized, double-blind, placebo-controlled phase III trial of apatinib in patients with chemotherapy-refractory advanced or metastatic adenocarcinoma of the stomach or gastroesophageal junction. J Clin Oncol 34: 1448-1454, 2016.

8. Pavlakis N, Sjoquist KM, Martin AJ, Tsobanis E, Yip S, Kang YK, Bang YJ, Alcindor T, O'Callaghan CJ, Burnell MJ, et al: Regorafenib for the treatment of advanced gastric cancer (INTEGRATE): A multinational placebo-controlled phase II Trial. J Clin Oncol 34: 2728-2735, 2016.

9. Kang YK, Rha SY, Tassone P, Barriuso J, Yu R, Szado T, Garg A and Bang YJ: A phase IIa dose-finding and safety study of first-line pertuzumab in combination with trastuzumab, capecitabine and cisplatin in patients with HER2-positive advanced gastric cancer. Br J Cancer 111: 660-666, 2014.

10. Fujitani K, Yang HK, Mizusawa J, Kim YW, Terashima M, Han SU, Iwasaki Y, Hyung WJ, Takagane A, Park DJ, et al: Gastrectomy plus chemotherapy versus chemotherapy alone for advanced gastric cancer with a single non-curable factor (REGATTA): A phase 3, randomised controlled trial. Lancet Oncol 17: 309-318, 2016.

11. Samarasam I, Chandran BS, Sitaram V, Perakath B, Nair A and Mathew G: Palliative gastrectomy in advanced gastric cancer: Is it worthwhile? ANZ J Surg 76: 60-63, 2006.

12. Hartgrink HH, Putter H, Klein Kranenbarg E, Bonenkamp JJ and van de Velde CJ; Dutch Gastric Cancer Group: Value of palliative resection in gastric cancer. Br J Surg 89: 1438-1443, 2002.

13. Medina-Franco H, Contreras-Saldívar A, Ramos-De La Medina A, Palacios-Sanchez P, Cortés-González R and Ugarte JA: Surgery for stage IV gastric cancer. Am J Surg 187: 543-546, 2004.

14. Fornaro L, Fanotto V, Musettini G, Uccello M, Rimassa L, Vivaldi C, Fontanella C, Leone F, Giampieri R, Rosati G, et al: Selecting patients for gastrectomy in metastatic esophago-gastric cancer: Clinics and pathology are not enough. Future Oncol 13: 2265-2275, 2017.

15. Sun J, Song Y, Wang Z, Chen X, Gao P, Xu Y, Zhou B and Xu H: Clinical significance of palliative gastrectomy on the survival of patients with incurable advanced gastric cancer: A systematic review and meta-analysis. BMC Cancer 13: 577, 2013.

16. Mohri Y, Tanaka K, Ohi M, Saigusa S, Yasuda H, Toiyama Y, Araki T, Inoue Y and Kusunoki M: Identification of prognostic factors and surgical indications for metastatic gastric cancer. BMC Cancer 14: 409, 2014.

17. Gadde R, Tamariz L, Hanna M, Avisar E, Livingstone A, Franceschi D and Yakoub D: Metastatic gastric cancer (MGC) patients: Can we improve survival by metastasectomy? A systematic review and meta-analysis. J Surg Oncol 112: 38-45, 2015. 
18. Rudloff U, Langan RC, Mullinax JE, Beane JD, Steinberg SM, Beresnev T, Webb C, Walker M, Toomey MA, Schrump D, et al: Impact of maximal cytoreductive surgery plus regional heated intraperitoneal chemotherapy (HIPEC) on outcome of patients with peritoneal carcinomatosis of gastric origin: Results of the GYMSSA trial. J Surg Oncol 110: 275-284, 2014.

19. Scartozzi M, Loretelli C, Galizia E, Mandolesi A, Pistelli M, Bittoni A, Giampieri R, Faloppi L, Bianconi M, Del Prete M, et al: Role of vascular endothelial growth factor (VEGF) and VEGF-R genotyping in guiding the metastatic process in pT4a resected gastric cancer patients. PLoS One 7: e38192, 2012.

20. Scartozzi M, Giampieri R, Loretelli C, Bittoni A, Mandolesi A, Faloppi L, Bianconi M, Del Prete M, Andrikou K, Bearzi I and Cascinu S: Tumor angiogenesis genotyping and efficacy of first-line chemotherapy in metastatic gastric cancer patients Pharmacogenomics 14: 1991-1998, 2013.

21. Iijima $\mathrm{Y}$, Akiyama $\mathrm{H}$, Atari M, Fukuhara M, Nakajima $\mathrm{Y}$, Kinosita $\mathrm{H}$ and Hidetaka U: Pulmonary resection for metastatic gastric cancer. Ann Thorac Cardiovasc Surg 22: 230-236, 2016.

22. Yoshida Y, Imakiire T, Yoneda S, Obuchi T, Inada K and Iwasaki A Ten cases of resected solitary pulmonary metastases arising from gastric cancer. Asian Cardiovasc Thorac Ann 22: 578-582, 2014.

23. Riihimäki M, Hemminki A, Sundquist K, Sundquist J and Hemminki K: Metastatic spread in patients with gastric cancer. Oncotarget 7: 52307-52316, 2016.

24. Van Raemdonck D: Pulmonary metastasectomy: Common practice but is it also best practice? Futur Oncol 11: 11-14, 2015

25. Treasure T, Fallowfield L and Lees B: Pulmonary metastasectomy in colorectal cancer: The PulMiCC trial. J Thorac Oncol 5 (6 Suppl 2): S203-S206, 2010.

26. Gonzalez $\mathrm{M}$ and Gervaz P: Risk factors for survival after lung metastasectomy in colorectal cancer patients: Systematic review and meta-analysis. Future Oncol 11 (Suppl 2): S31-S33, 2015.

27. Cancer Genome Atlas Research Network: Comprehensive molecular characterization of gastric adenocarcinoma. Nature 513: 202-209, 2014

28. Lauren P: The two histological main types of gastric carcinoma: Diffuse and so-called intestinal-type carcinoma. An attempt at a histo-clinical classification. Acta Pathol Microbiol Scand 64: 31-49, 1965.

29. Chen YC, Fang WL, Wang RF, Liu CA, Yang MH, Lo SS Wu CW, Li AF, Shyr YM and Huang KH: Clinicopathological variation of lauren classification in gastric cancer. Pathol Oncol Res 22: 197-202, 2016.
30. Schwartz LH, Litière S, de Vries E, Ford R, Gwyther S, Mandrekar S, Shankar L, Bogaerts J, Chen A, Dancey J, et al: RECIST 1.1-update and clarification: From the RECIST committee. Eur J Cancer 62: 132-137, 2016.

31. Bittoni A, Scartozzi M, Giampieri R, Faloppi L, Bianconi M, Mandolesi A, Del Prete M, Pistelli M, Cecchini L, Bearzi I and Cascinu S: Clinical evidence for three distinct gastric cancer subtypes: Time for a new approach. PLoS One 8: e78544, 2013.

32. Petrelli F, Berenato R, Turati L, Mennitto A, Steccanella F, Caporale M, Dellera P, de Braud F, Pezzica E, Di Bartolomeao M, et al: Prognostic value of diffuse versus intestinal histotype in patients with gastric cancer: A systematic review and meta-analysis. J Gastrointest Oncol 8: 148-163, 2017.

33. Fanotto V, Cordio S, Pasquini G, Fontanella C, Rimassa L, Leone F, Rosati G, Santini D, Giampieri R, Di Donato S, et al: Prognostic factors in 868 advanced gastric cancer patients treated with second-line chemotherapy in the real world. Gastric Cancer 20: 825-833, 2017.

34. Brandi G, Derenzini E, Falcone A, Masi G, Loupakis F, Pietrabissa A, Pinna AD, Ercolani G, Pantaleo MA, Di Girolamo S, et al: Adjuvant systemic chemotherapy after putative curative resection of colorectal liver and lung metastases. Clin Colorectal Cancer 12: 188-194, 2013.

35. Aurello P, Petrucciani N, Giulitti D, Campanella L, D'Angelo F and Ramacciato G: Pulmonary metastases from gastric cancer: Is there any indication for lung metastasectomy? A systematic review. Med Oncol 33: 9, 2016.

36. Shiono S, Sato T, Horio H, Chida M, Matsuguma H, Ozeki Y, Nakajima $\mathbf{J}$ and Okumura S; Metastatic Lung Tumor Study Group of Japan: Outcomes and prognostic factors of survival after pulmonary resection for metastatic gastric cancer. Eur J Cardiothorac Surg 43: e13-e16, 2013.

37. Bittoni A, Del Prete M, Scartozzi M, Pistelli M, Giampieri R, Faloppi L, Bianconi M and Cascinu S: Three vs. two drugs first-line chemotherapy regimen in advanced gastric cancer patients: A retrospective analysis. Springerplus 4: 743, 2015.

38. Zieliński M, Ochman M, Głowacki J and Kozielski J: Pulmonary lesions in the course of gastric cancer-two cases of Bard's syndrome. Pneumonol Alergol Pol 84: 33-37, 2016.

This work is licensed under a Creative Commons Attribution-NonCommercial-NoDerivatives 4.0 International (CC BY-NC-ND 4.0) License. 